\title{
The role of endothelin-1 and nitric oxide affecting endothelial dysfunction in the etiopathogenesis of ischaemic heart disease
}

\author{
Arpita Suri ${ }^{1, *}$, Ritu Singh ${ }^{2}$, Sanjay Tyagi ${ }^{3}$, J. Bhattacharjee $^{4}$
}

\begin{abstract}
${ }^{\mathbf{1}}$ Assistant Professor, Dept. of Biochemistry, SGT Medical college, Hospital and Research Institute, Gurugram, Haryana, ${ }^{2}$ Director and Professor, Dept. of Biochemistry, Lady Hardinge Medical College, New Delhi, ${ }^{3}$ Director, Professor and HOD, Dept. of Cardiology, G B Pant Institute of Post Graduate Medical Education and Research, New Delhi, ${ }^{4}$ Additional Director General, Lady Hardinge Medical College, New Delhi, India
\end{abstract}

*Corresponding Author: Arpita Suri

Email: arpita.lhmc@gmail.com

Received: $18^{\text {th }}$ July, 2018

Accepted: $25^{\text {th }}$ September, 2018

\begin{abstract}
Introduction: Endothelial dysfunction is an imbalance in bioavailability of endothelium-derived relaxing (Nitric Oxide) and contracting factors (Endothelin). Studies have shown that NO has antiatherogenic property as it inhibits vascular smooth muscle contraction and proliferation, platelet aggregation and leukocyte adhesion. On the other hand, Endothelin-1 causes proliferation and vasoconstriction of myocytes resulting in elevated vascular tone thereby contributing to endothelial dysfunction. This dysfunction is a well-established response to cardiovascular risk factors and precedes the development of atherosclerosis. As a result, status of an endothelial dysfunction may reflect the propensity to develop atherosclerotic disease and may serve as a marker of an unfavourable cardiovascular prognosis.

Materials and Methods: 50 cases of angiographically proven ischemic heart disease and 50 age and sex matched patients with no evidence of ischaemic heart disease on angiography were selected from G.B Pant Hospital, New Delhi, India. Serum levels of Endothelin were estimated by sandwich ELISA and nitric oxide levels by modified Griess reaction.

Results: Serum levels of Endothelin were raised while nitric oxide levels were significantly reduced $(p=0.049)$ in the cases as compared to controls. The mean plasma nitric oxide level in the study group (cases) was $23.51 \pm 13.48 \mu \mathrm{M}$ and in the control group was $29.35 \pm 15.73 \mu \mathrm{M}$. The difference between the two was significant $(\mathrm{p}=0.049 *)$. The mean plasma endothelin level in the study group was $3.04 \pm 1.37 \mathrm{fmol} / \mathrm{ml}$ and in the control group was $2.95 \pm 1.70 \mathrm{fmol} / \mathrm{ml}$. The difference between the two was not significant $(\mathrm{p}=0.764)$.

Conclusion: Higher Endothelin-1 and lower NO levels in cases suggest their role in causing endothelial dysfunction in ischemic heart disease patients. However, larger sample size is required to assess the role of Endothelin as a biomarker of endothelial dysfunction in patients of ischemic heart disease.
\end{abstract}

Keywords: Endothelial dysfunction, Endothelin-1, Nitric oxide, Ischemic heart disease, Atherosclerosis.

\section{Introduction}

During the last two decades, it has been shown that the vascular endothelium is an active paracrine, endocrine and autocrine organ that is indispensable for the regulation of vascular tone and the maintenance of vascular homeostasis. Anderson et $\mathrm{al}^{1}$ proposed endothelial dysfunction represents a key early step in the development of atheromatous plaque, its progression and the occurrence of atherosclerotic complications.

In 1992, Lerman and Burnett ${ }^{2}$ showed that endothelial dysfunction is characterized by reduction of the bioavailability of vasodilators, particularly nitric oxide (NO), and/or an increase in endothelium-derived contracting factors. The resulting imbalance leads to an impairment of endothelium-dependent vasodilation, a characteristic of endothelial dysfunction. Endothelial dysfunction also comprises of endothelial activation, which is characterized by a proinflammatory, proliferative, and procoagulatory states that favor all stages of atherogenesis. This results in a chronic inflammatory process accompanied by a loss of antithrombotic factors and an increase in vasoconstrictor and prothrombotic products, therefore elevating risk of cardiovascular events. ${ }^{3}$

Endothelial cells are able to synthesize and secrete a broad spectrum of anti-atherosclerotic substances, the most important of which is nitric oxide (NO). It is a gas that is generated from the metabolism of L-arginine by endothelial NO synthase (eNOS), constitutively expressed in endothelial cells. ${ }^{4}$

Nitric oxide (NO) also called endothelium derived relaxing factor (EDRF)-is one of the few gaseous signaling molecules known produced by endothelium. It stimulates guanyl cyclase and causes vasodilation by dephosphorylating myosin light chains of smooth muscle cell. It contributes to vessel homeostasis by inhibiting vascular smooth muscle contraction and proliferation, platelet aggregation and leukocyte adhesion. It is also generated by phagocytes (monocytes, macrophages, and neutrophils) as part of the human immune response. Phagocytes are armed with inducible nitric oxide synthase (iNOS), which is activated by interferon-gamma (IFN- $\gamma$ ) and tumor necrosis factor- $\alpha$ (TNF- $\alpha)$. It damages the DNA of foreign pathogen by producing free radicles. Therefore 
low levels of NO are associated with endothelial dysfunction(a state in which endothelium is associated with reduced anticoagulant properties as well as increased adhesion molecule expression, cytokine release and reactive oxygen species production), which is key event in development of atherosclerosis.

Under physiologic conditions, endothelial stimulation induces the production and release of NO, which diffuses to surrounding tissue and cells. It exerts its cardiovascular protective role by relaxing mediasmooth muscle cells, preventing leukocyte adhesion and migration into the arterial wall, muscle cell proliferation, platelet adhesion and aggregation and adhesion molecule expression ${ }^{5}$. The fundamental feature of endothelial dysfunction is the impaired NO bioavailability. This can be the consequence of either a reduced production by eNOS or, more frequently, an increased breakdown by reactive oxygen species (ROS). ${ }^{5}$ In the presence of impaired NO bioavailability, the endothelium implements various physiological pathways in the attempt to compensate for NO deficiency. Endothelium dependent vasodilation is also present by the production and release of endothelium derived vasodilators other than NO, such as prostanoids and other endothelium-derived hyperpolarizing factors. Excessive production of ROS destroys endogenous antioxidant defense mechanism and oxidize biological macromolecules, such as DNA, protein, carbohydrates, and lipids. This condition has commonly been referred to as oxidant stress.

\section{Endothelins}

Endothelins, a group of polypeptides, assist in vasomotor activity (transitory vasodilation and sustained vasoconstriction), proliferation of smooth muscle cells and hormone production. There are three endothelins: endothelin-1 (ET-1), endothelin-2 (ET-2), and endothelin-3 (ET-3). Each bind with three types of endothelin receptors: endothelin $\mathrm{A}\left(\mathrm{ET}_{\mathrm{A}}\right)$, endothelin $\mathrm{B}$ $\left(\mathrm{ET}_{\mathrm{B}}\right)$, and endothelin $\mathrm{C}\left(\mathrm{ET}_{\mathrm{C}}\right) . \mathrm{ET}_{\mathrm{A}}$ receptors are found in vascular smooth muscle tissue and cause vasoconstriction. $\mathrm{ET}_{\mathrm{B}}$ are located in the lining of the blood vessel wall and are associated with the release of vasodilators nitric oxide and prostacyclin. The role of $\mathrm{ET}_{\mathrm{C}}$ is still being researched.

Endothelin-1(a 21 amino acid peptide) is the most potent vasoconstricting agent identified and was originally isolated and characterized from the culture media of aortic endothelial cells. It is released from vascular endothelial cells, smooth muscle cells in the blood vessels, and macrophages. Under normal conditions, ET-1 is not activated. However, the production of ET-1 can be stimulated by angiotensin II (Ang II), thrombin, and transforming growth factor beta. Ang II is a protein that causes blood vessels to constrict, which increases resistance and the arterial pressure. The production of ET-1 is also increased by an increase in intracellular $\mathrm{Ca}^{2+6}$.
ET-1 is an important peptide because of its ability to initiate and maintain vasoconstriction for long term. In 2000, Fan et $\mathrm{al}^{7}$ proposed that it increases expression of endothelial adhesion molecules, acts as chemotactic agent for monocytes, and stimulates migration and proliferation of vascular smooth muscle cells.

Increased levels of ET-1 have been found in patients with atherosclerotic lesions and having risk factors for plaque development. Hypercholesterolemia, a risk factor for cardiovascular disease also affects ET1 , hereby increasing the adhesion of platelets, monocytes and migration of vascular smooth muscle cells. Oxidized LDL also increases the production of preproendothelin-1 mRNA which triggers the release of ET-1 from endothelial cells. In the pathogenesis of atherosclerosis, stimulated endothelial cells secrete endothelin and macrophage colony-stimulating factor (MCSF) which attract macrophages to a plaque site. Here, Angiotensin II, acting through the type 1 angiotensin receptor, causes macrophages and circulating platelets to adhere to the intima of the arteries. ET-1, which has mitogenic properties, stimulates fibroblast and smooth muscle cells proliferation with the aid of intracellular reactive oxygen species and mitogen-activated protein kinases. ${ }^{8}$ These causes atheromatous plaque formation. ${ }^{9}$ Hence, these correlations indicate a link between ET-1, atherosclerosis, and coronary artery disease. ${ }^{11}$

\section{Materials and Methods}

The study was carried out jointly in the department of Biochemistry, Lady Hardinge Medical College and in the department of Cardiology, G.B Pant Hospital.100 subjects were enrolled into the study during November 2011-April 2013, with informed consent. The Study group consisting of 50 patients of age 35 years and above with angiographically proven ischemic heart disease ( $>50 \%$ obstruction in one of the major epicardial arteries) and 50 age and sex matched controls with no evidence of IHD on angiography.

Detailed history was obtained from all the subjects and clinical examination was done. After an overnight fasting, venous blood samples were collected under sterile conditions and processed immediately for the routine biochemical investigations, hemogram and lipid profile. For special investigations, the plasma samples were stored at $-20^{\circ} \mathrm{C}$ till subsequent analysis. All the subjects underwent the same protocol.

Special investigations like Endothelin was estimated by ELISA kit from Biomedica gruppe (Austria) and estimation of Nitric oxide (NO) was done by modified Griess Reaction. ${ }^{10}$

\section{Statistical Analysis}

It was done by using SPSS (stastitical package for social sciences). All the data was expressed as mean \pm SD $/ \pm$ SE of mean. The $p$ value of $<0.05$ was considered as significant. The data obtained will be 
compared between two groups by student t-test. Pearson's correlation coefficient will be applied for correlation between two quantitative variables.

\section{Results}

The mean plasma nitric oxide level in the study group (cases) was $23.51 \pm 13.48 \mu \mathrm{M}$ and in the control group was $29.35 \pm 15.73 \mu \mathrm{M}$. The difference between the two was significant $\left(\mathrm{p}=0.049^{*}\right)$. The mean plasma endothelin level in the study group was $3.04 \pm 1.37 \mathrm{fmol} / \mathrm{ml}$ and in the control group was $2.95 \pm 1.70 \mathrm{fmol} / \mathrm{ml}$. The difference between the two was not significant $(\mathrm{p}=0.764)$. A negative correlation was found between endothelin with nitric oxide with $\mathrm{r}=$ 0.098 which was not significant.

Table 1: Serum endothelin level in study and control group

\begin{tabular}{|l|c|c|c|c|}
\hline $\begin{array}{c}\text { Study } \\
\text { Group } \\
\text { (N=50) }\end{array}$ & MEAN & S.D & S.E.M & P Value \\
\hline Case & 3.04 & 1.37 & 0.193 & \\
\hline Control & 2.95 & 1.70 & 0.241 & 0.764 \\
\hline
\end{tabular}

${ }^{*} p$ value $\leq 0.05$ is considered statistically significant.

The mean plasma endothelin level in the study group was $3.04 \pm 1.37 \mathrm{fmol} / \mathrm{ml}$ and in the control group was $2.95 \pm 1.70 \mathrm{fmol} / \mathrm{ml}$. The difference between the two was not significant $(p=0.764)$.

Table 2: Plasma nitric oxide level in study and control group

\begin{tabular}{|l|c|c|c|c|}
\hline $\begin{array}{c}\text { Study } \\
\text { Group(n=50) }\end{array}$ & MEAN & S.D & S.E.M & P Value \\
\hline Case & 23.51 & 13.48 & 1.90 & $0.049^{*}$ \\
\hline Controls & 29.35 & 15.73 & 2.22 & \\
\hline
\end{tabular}

$* p$ value $\leq 0.05$ is considered statistically significant.

The mean plasma nitric oxide level in the study group (cases) was $23.51 \pm 13.48 \mu \mathrm{M}$ and in the control group was $29.35 \pm 15.73 \mu \mathrm{M}$. The difference between the two was significant $\left(\mathrm{p}=0.049^{*}\right)$.

\section{Discussion}

The mean plasma Endothelin levels were higher in cases group $(3.04 \pm 1.37 \mathrm{fmol} / \mathrm{ml})$ than controls $(2.95 \pm 1.70 \mathrm{fmol} / \mathrm{ml})$ suggestive role of vasoconstrictive effect of endothelin in IHD patients. Sainani et $\mathrm{al}^{11}$ showed in south Asian population high levels of endothelin was responsible for endothelial cell dysfunction but only in hypertensives. Salomone et al showed raised ET-1 levels in IHD and correlated it with severity of the disease. ${ }^{12}$ A large body of evidence has accumulated, indicating that the endothelins are involved in vascular dysfunction by modulating nitric oxide levels as proven by my study though statistically not significant. A negative correlation was found between endothelin with nitric oxide with $\mathrm{r}=-0.098$ which was not significant. Luscher et $\mathrm{al}^{4}$ also proved that $\mathrm{ET}_{\mathrm{A}}$ antagonist increased the amount of nitric oxide present and decreased the endothelial dysfunction but their study was done in Caucasian population. The mean plasma nitric oxide level in the study group (cases) was $23.51 \pm 13.48 \mu \mathrm{M}$ and in the control group was $29.35 \pm 15.73 \mu \mathrm{M}$. The difference between the two was significant $(\mathrm{p}=0.049 *)$. The mean plasma NO levels was lower in case group $23.51 \pm 13.48 \mu \mathrm{M}$ as compared to controls $29.35 \pm 15.73 \mu \mathrm{M}$ which points to endothelial dysfunction in IHD patients. In addition, Flammer et $\mathrm{al}^{13}$ and Lüscher et al ${ }^{14}$ have also postulated that endothelial dysfunction is characterized by an impaired NO bioavailability and it occurs early in the development of atherosclerosis. Thus, my study agrees with the antiatherogenic hypothesis of NO in IHD.

\section{Conclusion}

Our study supports that endothelial dysfunction characterized by rise in vasoconstrictive factors like Endothelin and a fall in vasodilators like nitric oxide is an important feature of IHD. These novel biomarkers of endothelial dysfunction need further evaluation in larger sample size for better non-invasive diagnosis of atherosclerosis.

\section{References}

1. Anderson TJ, Gerhard MD, Meredith IT. Systemic nature of endothelial dysfunction in atherosclerosis. Am J Cardiol. 1995;75:71-4.

2. Lerman A, Burnett JC. Intact and altered endothelium in regulation of vasomotion. Circulation. 1992;86(3):12-9.

3. Bonetti PO, Lerman LO, Lerman A. Endothelial dysfunction a marker of atherosclerotic risk. Arterioscler Thromb Vasc Biol. 2003;23(2):168-75.

4. Luscher TF, Vanhoutte PM. The Endothelium: Modulator of Cardiovascular Function. Boca Raton, FL, CRC Press 1990.

5. Taddei S, Ghiadoni L, Virdis A, Versari D, Salvetti A. Mechanisms of endothelial dysfunction: clinical significance and preventive non-pharmacological therapeutic strategies. Curr Pharm Des. 2003;9:2385402.

6. Luscher TF, RR Wenzel. "Endothelin and Endothelin Antagonists: pharmacology and clinical implications." Agents Actions Suppl. 1995;45:237-53.

7. Fan J, Unoki H, Iwasa S, Watanabe T. Role of endothelin-1 in atherosclerosis. Ann N Y Acad Sci. 2000;902:84-93.

8. Cheng CM, Hong HJ, Liu JC, Shih NL, Juan SH, Loh SH et al. Crucial Role of Extracellular Signal-Regulated Kinase Pathway in Reactive Oxygen Species-Mediated Endothelin-1 Gene Expression Induced by Endothelin-1 in Rat Cardiac Fibroblasts. The American Society for Pharmacology and Experimental

Therapeutics. 2003;63:1002-11.

9. Touyz RM, Chen X, Tabet F, Yao G, He G, Quinn MT et al. Expression of a functionally active gp91phoxcontaining neutrophil-type $\mathrm{NAD}(\mathrm{P}) \mathrm{H}$ oxidase in smooth muscle cells from human resistance arteries: regulation by angiotensin II. Circ Res. 2002;90:1205-13. 
10. Mathew F, Glenda J, Jack L. Quantification of nitric and nitrate in extracellular fluids. Metho Enzymol. 1996;268:237-46.

11. Sainani GS, Maru VG. Role of Endothelial Cell Dysfunction in Essential Hypertension. JAPI. 2004;52:966-9.

12. Salomone OA, Elliott PM, Calvino R, Holt D, Kaski JC. Plasma immunoreactive endothelin concentration correlates with severity of coronary artery disease in patients with stable angina pectoris and normal ventricular function. J Am Coll Cardiol. 1996;28:14-9.

13. Flammer AJ, Luscher TF. Three decades of endothelium research: from the detection of nitric oxide to the everyday implementation of endothelial function measurements in cardiovascular diseases. Swiss Med Wkly. 2010;140:w13122.

14. Lüscher TF, Vanhoutte PM. The Endothelium: Modulator of Cardiovascular Function. Boca Raton: CRC Press 1990.

How to cite this article: Suri A, Singh R, Tyagi $\mathrm{S}$, Bhattacharjee J. The role of endothelin-1 and nitric oxide affecting endothelial dysfunction in the etiopathogenesis of ischaemic heart disease. Int J Clin Biochem Res. 2018;5(4):537-540. 